\title{
Investigation of Microbiological Methods to Estimating Individual Caries Risk: Evaluation of Sampling Methods and Materials.
}

\author{
Tomotake Neta ${ }^{1}$, Ritsuko Inokuchi', Noriko Shinozaki-Kuwahara ${ }^{1}$, Yoshiharu Kouno ${ }^{2}$, Takuji Ikemi ${ }^{2}$ and \\ Kazuo Fukushima ${ }^{1}$
}

Departments of 'Microbiology and ${ }^{2}$ Operative Dentistry, Nihon University School of Dentistry at Matsudo, Matsudo, Chiba 2718587, Japan.

Correspondence to:

Dr.Kazuo Fukushima

E-mail: kfuku@mascat.nihon-u.ac.jp

Key words

mutans streptococci, dental plaque, caries-

risk

\begin{abstract}
Stimulated-saliva, swabbing-plaque and brushing-plaque samples were successively collected from five volunteers four times over a seven-day period and the number of total streptococci (TS) and mutans streptococci (MS) in each sample were determined using selective media. The percentages of MS in TS (MS\%) of the brushing-plaque and the stimulated saliva, but not the swabbingplaque, were kept almost constant through a sampling period, and the ratio of mean MS\% in both samples significantly varied among subjects. These findings suggest that plaque sample recovered by brushing treatment for $1 \mathrm{~min}$ is superior to stimulated whole saliva as a sampling material for assessment of individual caries risk.
\end{abstract}

\section{Introduction}

Mutans streptococci are the principal etiological agents of dental caries. The degree of colonization of these organisms correlates with the prevalence of dental caries in human and experimental animals. An important virulence factor of mutans streptococci promoting caries development is its ability to colonize firmly on the tooth surface in the presence of sucrose and produce the cariogenic biofilm in situ (1-3). Of seven species of mutans streptococci, Streptococcus mutans and Streptococcus sobrinus are isolated as the predominant species from human saliva and dental plaque $(4,5)$. These organisms show genetic (6-8), biochemic $(5,8-11)$ and pathogenic $(11,12)$ polymorphisms, and their infectious levels are largely different for each oral cavity $(1,2,13,14)$. Therefore, each individual oral cavity possesses a different virulence potential (caries risk) $(1,2)$. Foreknowledge of the caries risk of individuals is essential for effective caries prevention. However, there is no definitive rapid method for estimation of individual caries risk. Thus, we have started basal studies to construct a useful microbiological method for rapid assessment of the individual caries risk. In this study, we describe a part of the experimental results concerning sampling methods and materials.

\section{Materials and Methods}

Subjects:

Five healthy human volunteers (A to E, aged 21 to 43 years) with detectable levels of mutans streptococci on the tooth surfaces participated in this study.

\section{Preparation of Oral Samples:}

In the late morning (about eleven o'clock) on alternate days (days 1, 3, 5 and 7), oral samples of stimulated saliva, swabbing-plaque and brushing-plaque were successively collected from each subject by the following methods. Saliva secreted over a period of 3 minutes, while being stimulated by biting paraffin gum was collected in a sterile bottle, icechilled, and used as a stimulated saliva sample. After the subject mouth-washed with drinking water, plaque was taken by scrubbing for $10 \mathrm{sec}$ with a sterile cotton swab the alveolar ridge and the buccal surfaces of first and second molars in the right side of the upper jaw. The cotton was then cut and dipped in $4 \mathrm{ml}$ ice-chilled Brain Heart Infusion (BHI) broth, and its sonicated suspension was used as a swabbing-plaque sample. A large portion of plaque on all teeth was then scraped off by vigorous brushing for $1 \mathrm{~min}$ with a sterile toothbrush and collected in a sterile bottle through a mouth rinse for $30 \mathrm{sec}$ with $5 \mathrm{ml}$ phosphate-buffered saline (PBS), ice-chilled, and used as a brushing-plaque sample. 


\section{Microbiological Analysis:}

Mitis-Salivarius agar (MS agar, Difco, MI, USA) and MS agar supplemented with $0.2 \mathrm{unit} / \mathrm{ml}$ Bacitracin and $15 \%$ sucrose (MSB agar) (15) were used as a selective medium for total streptococci and mutans streptococci, respectively. Within $3 \mathrm{hr}$ after sampling, the oral samples were dispersed by sonication treatment $(50 \mathrm{~W}, 20 \mathrm{sec})$ using an ultrasonic apparatus (5202 Type, Otake Works, Tokyo), serially diluted with chilled BHI broth, and inoculated on the selective media using a spiral plating system (Model-D, Gunze Sangyo, Ink., Tokyo). After anaerobic culture for $48 \mathrm{hr}$, total streptococci colonies on the MS agar plates and mutans streptococci colonies on the MSB agar plates were counted. S. mutans colonies and $S$. sobrinus colonies could be distinguished from their colonial morphology on MSB agar and BHI agar plates.

\section{Results and Discussion}

From each of five healthy subjects, three oral samples of stimulated saliva, swabbing-plaque and brushing-plaque were successively collected 4 times over a seven- day period, and the number of total streptococci and mutans streptococci in each sample was determined by the cultivation method using selective media, MS agar and MSB agar. When the colonies on MSB agar plates were evaluated using a stereomicroscope, S. mutans was detected in all samples from the five subjects while $S$. sobrinus was detected in samples from subject E. From the counts of total streptococci (TS) and mutans streptococci (MS), the percentages of MS in TS (MS\%) were calculated and compared. The results are summarized in tables 1 and 2 . Mean MS\% (average \pm SD) of the four stimulated saliva samples from each subject (A to E) were $1.47 \pm 0.31,1.08$ $\pm 0.10,0.43 \pm 0.02,0.27 \pm 0.02$ and $0.20 \pm 0.01$, respectively. Those of the four swabbing-plaque samples were $2.46 \pm 1.65,0.15 \pm 0.14,0.06 \pm 0.06,1.27 \pm 1.18$

Table 1. Percentages of mutans streptococci (MS) in total streptococci (TS) of stimulatedsaliva, swabbing-plaque and brushing-plaque samples collected from five subjects

\begin{tabular}{|c|c|c|c|c|}
\hline \multirow{2}{*}{ Subject } & \multirow{2}{*}{$\begin{array}{l}\text { Sampling } \\
\text { (day) }\end{array}$} & \multicolumn{3}{|c|}{$\mathrm{MS} \%$} \\
\hline & & Stimulated saliva & Swabbing plaque & Brushing plaque \\
\hline \multirow[t]{4}{*}{ A } & 1 & 1.53 & 4.57 & 3.16 \\
\hline & 3 & 1.18 & 1.01 & 2.44 \\
\hline & 5 & 1.88 & 1.29 & 2.98 \\
\hline & 7 & 1.30 & 2.96 & 3.11 \\
\hline \multirow[t]{4}{*}{ B } & 1 & 1.10 & 0.02 & 0.20 \\
\hline & 3 & 0.97 & 0.21 & 0.19 \\
\hline & 5 & 1.05 & 0.05 & 0.22 \\
\hline & 7 & 1.20 & 0.32 & 0.28 \\
\hline \multirow[t]{4}{*}{$\mathrm{C}$} & 1 & 0.43 & 0.02 & 0.48 \\
\hline & 3 & 0.45 & 0.14 & 0.65 \\
\hline & 5 & 0.42 & 0.02 & 0.55 \\
\hline & 7 & 0.41 & 0.04 & 0.47 \\
\hline \multirow[t]{4}{*}{$\mathrm{D}$} & 1 & 0.30 & 0.53 & 1.77 \\
\hline & 3 & 0.27 & 0.46 & 2.01 \\
\hline & 5 & 0.27 & 2.99 & 1.87 \\
\hline & 7 & 0.25 & 1.08 & 1.94 \\
\hline \multirow[t]{4}{*}{$\mathrm{E}$} & 1 & 0.19 & 1.60 & 2.08 \\
\hline & 3 & 0.21 & 0.19 & 1.88 \\
\hline & 5 & 0.18 & 0.85 & 2.22 \\
\hline & 7 & 0.20 & 1.03 & 1.59 \\
\hline
\end{tabular}


Table 2. Comparison of the mean MS\% values of stimulated-saliva and brushing-plaque samples collected from five subjects

\begin{tabular}{cccc}
\hline \multirow{2}{*}{ Subject } & \multicolumn{2}{c}{ MS\% (Ave. \pm SD) } & $\begin{array}{c}\text { Plaque MS\% } \\
\text { /Saliva MS\% }\end{array}$ \\
\cline { 2 - 3 } & Stimulated saliva & Brushing-plaque & \\
\hline A & $1.47 \pm 0.31$ & $2.92 \pm 0.33$ & $1.98 \pm 0.33$ \\
B & $1.08 \pm 0.10$ & $0.22 \pm 0.04$ & $0.21 \pm 0.02$ \\
C & $0.43 \pm 0.02$ & $0.54 \pm 0.08$ & $1.26 \pm 0.15$ \\
D & $0.27 \pm 0.02$ & $1.90 \pm 0.10$ & $6.96 \pm 0.81$ \\
E & $0.20 \pm 0.01$ & $1.94 \pm 0.27$ & $9.96 \pm 1.97$ \\
\hline
\end{tabular}

and $0.92 \pm 0.58$, respectively. And, those of the four brushing-plaque samples were $2.92 \pm 0.33,0.22 \pm 0.04$, $0.54 \pm 0.08,1.90 \pm 0.10$ and $1.94 \pm 0.27$, respectively. These results indicate that individual MS\% values of brushing-plaque samples, as well as those of stimulated saliva samples, were kept almost constant through the sampling period while those of the swabbing-plaque samples varied considerably. The method to prepare the brushingplaque samples is very simple and easy although it cannot recover selectively only plaque bacteria. This sampling method may be useful as a means for assessment of individual caries risk and for epidemiological study.

Also, when the mean MS\% values in the whole saliva samples and the brushing-plaque samples from an individual were compared, significant variations were observed among subjects. As table 2 shows, the mean MS\% value of the brushing-plaque from subjects $\mathrm{A}, \mathrm{D}$ and $\mathrm{E}$ were higher, around 2, 7 and 10 times, respectively, than those of the whole stimulated saliva. In contrast, the value of the whole stimulated saliva from subject B was higher, around fivefold than that of the brushing-plaque. Only for subject $\mathrm{C}$ were the values of both samples almost the same. These findings strongly suggest that whole stimulated saliva is not suitable as a sample for the caries risk assessment of individuals and that a plaque sample is essential for it because the tooth surfaces constitute the natural habitat of mutans streptococci. Many epidemiological studies have shown positive correlations between levels of mutans streptococci in saliva and plaque (16-18), and the enumeration of these cariogenic bacteria therefore have often been done with stimulated saliva samples, which are easier to obtain. However, data in those epidemiological studies also indicate that an individual ratio of MS levels in saliva and plaque significantly differs among subjects.

An epidemiological study to clarify the relationship between MS\% values in brushing-plaque samples and caries experience is in progress.

\section{Acknowledements}

This study was partly supported by research grants from Nihon University and the Ministry of Education, Culture Sports Science, and Technology to promote research between academia and industries, and a Health Science research grant.

\section{References}

1. Hamada S, Slade HD: Biology, immunology and cariogenisity of Streptococcus mutans. Microbiol Rev, 44:331-384, 1980.

2. Loeshe WJ: Role of Streptococcus mutans in human dental decay. Microbiol Rev, 50:353-380, 1986.

3. Kuramitsu HK: Virulence factors of mutans streptococci: role of molecular genetics. Crit Rev Oral Biol Med, 4:159-176, 1993.

4. Coykendall AL: Classification and identification of the viridans streptococci. Clin Microbiol Rev, 2:315-328, 1989.

5. Masuda N, Tsumi N, Sobue S, Hamada S: Longitudinal survey of the distribution of various serotypes of Streptococcus mutans in Infants. J Clin Microbiol, 10:497-502, 1979.

6. Saarela M, Alalussua S, Takei T, Asikainen S: Genetic diversity within isolates of mutans streptococci recognized by an rRNA gene probe. J Clin Microbiol, 31:584-587, 1993.

7. Caufield PW, Walker TM: Genetic diversity within Streptococcus mutans evident from chromosomal DNA restriction fragment polymorphisms. J Clin Microbiol, 27:274-278, 1989.

8. Harper DS, Loesche WJ: Effect of pH upon sucrose and glucose catabolism by the various genogroups of Streptococcus mutans. J Dent Res, 62:526-531, 1983.

9. Tomita Y, Zhu X, Ochiai K, Namiki Y, Okada T, Ikemi T, Fukushima K: Evaluation of three individual 
glucosyltransferases produced by Streptococcus mutans using monoclonal antibodies. FEMS Microbiol Lett, 145:427-432, 1997.

10. Alaluusua S, Gronroos L, Zhu X, Saarela M, Matto J, Asikainen S, Fukushima K: Production of Glucosyltransferases by clinical mutans streptococcal isolates as determined by semiquantitative cross-dot assay. Archs oral Biol, 42:417-422, 1997.

11. Macpherson LMD, MacFarlane TW, Geddes DAM, Stephen KW: Assessment of the cariogenic potential of Streptococcus mutans strains and its relationship to in vivo caries experience. Oral Microbiol Immunol, 7:142-147, 1992.

12. de Soet JJ, van Loveren C, Lammens AJ, Pavicic MJ, Homburg CH, ten Cate JM, de Graff J: Differences in cariogenicity between fresh isolates of Streptococcus sobrinus and Streptococcus mutans. Caries Res, 25:116-122, 1991.
13. Togelius J, Bratthal D: Frequency of the bacterium Streptococcus mutans in the saliva of selected human populations. Archs oral Biol, 27:113-116, 1982.

14. Kohler B, Bjarnason S: Mutans streptococci, lactobacilli and caries prevalence in 11- and 12-year-old Icelandic children. Community Dent Oral Epidemiol, 15:332-335, 1987.

15. Gold OJ, Jordan HV, van Houte J: A selective medium for Streptococcus mutans. Archs oral Biol, 18:1357-1364, 1973.

16. Keene HJ: Sampling of cariogenic microorganisms in human populations. Oral Microbiol Immunol, 1:7-12, 1986.

17. Schaeken MJM, Creugere TJ, van der Hoeven JS: Relationship between dental plaque indices and bacteria in dental plaque and those in saliva. J Dent Res, 66:1499-1502, 1987.

18. Mundorff SA, Eisenberg AD, Leverett DH, Espeland MA, Proskin HM: Correlations between numbers of microflora in plaque and saliva. Caries Res, 24:312-317, 1990. 